\title{
Violencia conyugal y dependencia afectiva: un estudio cualitativo en población masculina
}

\section{Marital violence and affective dependence: a qualitative study in male population}

DOI: $10.18270 /$ chps..v78i1.2854

Recibido: 16-11-2018 Aceptado: 14-03-2019 https://revistas.usb.edu.co/index.php/IJPR

\section{* Katherine Díaz}

Psicóloga, Universidad Cooperativa de Colombia, Sede Pereira, Colombia,

Email:katherine.diazp@campusucc.edu.co

\section{* Verónica García-Cardona}

Psicóloga, Universidad Cooperativa de Colombia, Sede Pereira, Colombia,

rosa.garciac@campusucc.edu.co

\section{* Sergio Humberto Barbosa Granados}

Mg. en Informática Aplicada a la Educación; Especialista en Práctica Pedagógica Universitaria; Psicólogo. Docente e Investigador, Programa de Psicología, Universidad Cooperativa de Colombia, Sede Pereira, Colombia Email: sergio.barbosag@campusucc.edu.co

Copyright: ๑2019.

La Revista Cuadernos Hispanoamericanos de Psicología proporciona acceso abierto a todos sus contenidos bajo los términos de la licencia creative commons Attribution-NonCommercial- NoDerivatives 4.0 International (CC BY-NCND 4.0)

Declaración de disponibilidad de datos: Todos los datos relevantes están dentro del artículo, así como los archivos de soporte de información.

Conflicto de intereses: Los autores han declarado que no hay conflicto de intereses

\begin{abstract}
Resumen
La violencia conyugal ha sido un fenómeno psicosocial de interés, además de ser una problemática de salud pública que ha generado grandes repercusiones tanto para quienes son víctimas, como para quienes les rodean. Este tipo de violencia también ha sido una problemática legitimada y estudiada con un enfoque especial hacia la mujer. La presente investigación es cualitativa- hermenéutica y se realizó a partir de escenas videográficas que representaron violencia conyugal hacia los hombres. Además, a través de entrevistas semiestructuradas se identificaron y comprendieron los significados que, sobre violencia conyugal, tenían seis hombres adscritos a la facultad de psicología de una universidad privada de Colombia a partir de la perspectiva sobre la dependencia afectiva. Lo anterior, con el fin de caracterizar la problemática y darle mayor visibilidad. Los resultados evidenciaron que los hombres significan la violencia conyugal hacia el hombre como acciones físicas que se dan entre pareja, comportamientos que se toleran con la finalidad de ser digno de la misma, como una consecuencia de la dependencia afectiva y/o como un comportamiento de dominancia que genera burlas. Más allá de los resultados esperados, también se pudo observar que la invisibilización de la violencia conyugal hacia el hombre tiene una alta relación con los patrones culturales que implica el género en una sociedad machista.
\end{abstract}

Palabras clave: Significados, violencia conyugal, dependencia afectiva, violencia de género, masculinidad, hegemonías culturales. 


\begin{abstract}
Conjugal violence has been a psychosocial phenomenon of interest, as well as a public health problem that has generated great repercussions both for those who are victims and those around them. In addition, it has been a problem legitimized and studied with a special focus on women. This is a qualitative-hermeneutic research developed through videographic scenes that represented conjugal violence towards men and through semi-structured interviews. The meanings on conjugal violence given by six men assigned to the psychology faculty of a private university of Colombia, were identified and understood from the perspective of affective dependence in order to characterize the problem, as well as giving it more visibility. The results showed that men mean conjugal violence towards men as physical actions that occur between partners, behaviors that are tolerated with the purpose of being worthy of the other, a consequence of affective dependence and/or as a behavior of dominance that generates teasing. Beyond the expected results, it was also observed that the invisibility of conjugal violence towards men is highly related to cultural patterns implied by genre in a sexist society.
\end{abstract}

Keywords: Meanings, conjugal violence, affective dependence, gender violence, masculinity, cultural hegemonies.

\title{
Introducción
}

El término violencia normalmente está representado por la carga de rabia y descarga agresiva en la conducta humana hacia el otro; a su vez, esta se encuentra asociada con sentimientos como el odio y las actitudes destructivas, donde cada uno de los participantes niega su responsabilidad de lo que ocurre y culpa al otro (Lessard et al., 2015; Perrone \& Nannini, 2007). Esto demuestra la incapacidad de reconocer la diferencia, la singularidad y los deseos de cada persona, así como la dificultad de aceptar y cumplir las reglas, lo cual trae consigo conductas violentas.

Entre los tipos de violencia se encuentra la violencia conyugal (VC), considerada como un tipo de violencia interpersonal, donde el comportamiento dentro de una relación íntima o de pareja, causa daño físico, psíquico o sexual. La VC incluye agresiones físicas (abofetear, golpear con los puños o con cualquier parte del cuerpo, golpes con objetos, empujones, estrangulamiento, entre otros), relaciones sexuales forzadas, otras formas de coacción sexual y maltrato psicológico, con diversos comportamientos dominantes hacia el otro (aislamiento de la familia y amistades, vigilar sus movimientos y restringir su acceso a la información o asistencia, entre otros), e incluso la muerte (Ansara \& Hindin, 2011; Heise \& García-Moreno 2013; World Health Organization, 2011).

Uno de los aspectos psicológicos que, por sus características, posiblemente coexista con la VC es la dependencia afectiva (DA). Ésta ha sido definida como un fenómeno disfuncional en el ámbito relacional e interpersonal, entendiéndose como una necesidad extrema de orden afectivo que la persona siente hacia su pareja (Castelló, 2005; Moral \& Sirvent, 2008). En las personas con DA, se ha podido encontrar una pérdida de identidad, que puede llegar hasta el punto de subordinarse con el fin de retener a la pareja (Schaeffer, 1998). También se ha analizado que la DA es un patrón de comportamiento que se desarrolla desde la infancia. En el mismo sentido, Bowlby (1980; 2003) afirma que los vínculos de apego se generan en los niños con la finalidad de satisfacer sus necesidades físicas y emocionales básicas; sin embargo, algunos llegan a generar conductas de apego; es decir, conductas destinadas a mantener proximidad con otro sujeto por medio de la amenaza o el reclamo constante. Según Castelló (2005), este tipo de comportamiento puede llegar a ser replicado en la adultez como pautas relacionales; estas pautas o esquemas tempranos son estables a lo largo de la vida y poseen un carácter resistente al cambio, hasta el punto de llevar a un individuo a distorsionar la información (Young, 1999).

Así mismo, se establece que la baja autoestima y la autoimagen negativa causan temor recurrente al abandono y el rechazo, lo cual dificulta la terminación de una relación, incluso si ésta se considera disfuncional (Ocampo \& Amar, 2011). En este orden de ideas, Branden (1995) señala 
que la autoestima es "la disposición a considerarse frente a los desafíos de la vida y sentirse merecedor de la felicidad. Tener una alta autoestima es sentirse apto para la vida, competente y merecedor; tener una baja autoestima es sentirse inapropiado para la vida" (p.1).

Los estudios que han indagado sobre el fenómeno de VC han encontrado una asociación entre el maltrato en las relaciones de pareja y las variables psicológicas, como características de la personalidad (Castelló, 2005; Dubugras \& Guevara, 2013; López, Moral, Díaz \& Cienfuegos, 2013; Moral \& Sirvent, 2008; Picó, Echeburúa \& Martínez, 2008; Preciado, Torres \& Rey, 2012). Las anteriores perspectivas apuntan a la existencia de un fenómeno de dependencia afectiva como consecuencia del dominio y la manipulación que se da en una pareja violenta (Aiquipa-Tello, 2015; Amor \& Echeburúa, 2010; Castelló, 2005; Hirigoyen, 2006; Villegas \& Sánchez, 2013). En ese sentido, algunos hallazgos sobre jóvenes y personas con dependencia afectiva han permitido descubrir que este fenómeno se asocia con insuficiencia en esquemas de autocontrol, conductas límites, relaciones de abuso y desconfianza (Lemos \& Londoño, 2006).

Frente a los antecedentes hallados alrededor de la problemática de VC hacia el género masculino, existe una diversidad frente a la caracterización o correlación del fenómeno con otras variables. Al respecto, una investigación realizada en Chile, denominada los hombres también sufren (RojasAndrade, Galleguillos, Miranda \& Valencia, 2013), concluye que las mujeres ejercen violencia psicológica sobre los hombres, a través de gritos e imposición de control constante. Cuando este tipo de conductas no logran tener el efecto esperado, surge la violencia física. En los discursos analizados, también se encontró que la baja autoestima y la dependencia afectiva estaban relacionadas con la tolerancia que poseen los hombres ante la situación de violencia ejercida por su pareja (Rojas-Andrade et al., 2013).

Por su parte, una investigación sobre la violencia de pareja contra el hombre en la ciudad de Cali determinó que la mayoría de los hombres que sufren esta problemática viven en unión libre; también se halló que la intolerancia y la celotipia son desencadenantes importantes de este tipo de violencia (Floyd-Aristizábal, Loaiza, Sierra, López \& Ricaurte, 2016). En el año 2017, se llevó a cabo un estudio en México, el cual analizó la dependencia emocional en el conflicto de pareja; se observó que los componentes de este tipo de dependencia se correlacionaron positivamente, aunque con intensidad débil, con el miedo a la soledad, lo cual podría aclarar por qué se mantiene en el tiempo el conflicto de pareja (Laca \& Mejía, 2017).

A su vez, otro estudio realizado con hombres que han sido violentados por la pareja en la ciudad de Baja California-México concluyó que existen evidencias de violencia por parte de la mujer hacia el hombre, situación que alienta a trabajar sobre esta problemática social real, pero poco convencional. Además, sugiere que se deben estudiar los principales factores de riesgo hallados en la investigación, tales como la condición migratoria y el hecho de haber sufrido algún tipo de violencia en la infancia. Así mismo, considera que se deben tener en cuenta factores como la edad, la educación y el hecho de desarrollar o no una actividad laboral. No obstante, se pone énfasis en la importancia de continuar estudiando la violencia creciente del hombre hacia la mujer (González \& Fernández, 2014).

Ahora bien, la violencia conyugal ha sido un fenómeno psicosocial legitimado y estudiado con un enfoque especial hacia la mujer, por lo que se han destacado más casos en donde el género femenino ha sido la principal víctima (Ravazzola, 1997; Duarte, Gómez \& Carrillo, 2010; Toldos, 2013). Sin embargo, en los últimos años se reconoce que los hombres también son víctimas de mujeres violentas, por lo que cada vez hay mayor interés por estudiar esta problemática (RojasAndrade et al., 2013; Saracostti, 2011; Zunino, 2011).

De acuerdo con lo anterior, es relevante seguir indagando el fenómeno de la violencia conyugal hacia el hombre, de tal forma que la investigación le dé más visibilidad a esta problemática que 
por tanto tiempo se ha mantenido oculta. En este mismo sentido, con relación a las características de la DA, la presente investigación puede brindar herramientas que caractericen el fenómeno desde una perspectiva psicológica. Esto permitirá que los hombres puedan reconocer cuándo están siendo víctimas de violencia conyugal y se genere una mayor exposición de casos, lo que hará posible una profundización efectiva acerca de las dinámicas que surgen en dicha situación.

\section{Método}

\section{Diseño}

Teniendo en cuenta que la experiencia humana acontece en un contexto histórico- cultural, donde las personas construyen significados a partir de dichas experiencias - significados que, según Bruner (1991), denotan sentido y referencia, además de develar las circunstancias en las cuales surgen las divergentes interpretaciones de la realidad (p.75)., la presente investigación optó por un diseño metodológico cualitativo, en donde se implementó el método hermenéutico para la comprensión de los significados sobre violencia conyugal hacia el hombre, debido a que " el acercamiento hermenéutico busca elucidar y hacer explícita nuestra comprensión práctica de las acciones humanas al proveer una interpretación de ellas. Es un acercamiento históricamente situado que considera la explicación, antes que nada, como el dar una versión que sea sensible en la manera como se dirige a los intereses y preocupaciones actuales, no como una búsqueda de leyes eternas y ahistóricas, y de estructuras formales" (Packer, 1985, p.17).

De esta manera, por medio de la narrativa de las experiencias individuales de los participantes, se logró identificar los significados que estos tenían sobre la violencia conyugal hacia el hombre, comprender el fenómeno a la luz de las características de la dependencia afectiva, así como caracterizarlo de manera que tenga mayor visibilidad. Lo anterior, teniendo en cuenta que, según Brunner (1991), Nuestra capacidad para contar nuestras experiencias en forma de narración no es sólo un juego de niños, sino también un instrumento para proporcionar significado que domina gran parte de la vida en una cultura.

\section{Participantes}

Los participantes del presente estudio fueron seis hombres entre los 20 y 40 años, estudiantes de séptimo, octavo y décimo semestre de la facultad de psicología de una universidad privada de Colombia, los cuales fueron elegidos por disponibilidad; es decir, fueron voluntarios. Cuatro de ellos tenían una relación de pareja heterosexual y eran monógamos; uno de ellos era soltero y el otro, polígamo; todos pertenecían a diferentes estratos socioeconómicos. Se desconocía si los participantes contaban con algún tipo de experiencia de violencia conyugal, pero fueron elegidos por tener presuntos conocimientos sobre la problemática dado que eran estudiantes de psicología. Lo anterior con la finalidad de develar lo que significaban respecto a la problemática, e identificar si, como hombres, se reconocían como víctimas de algún tipo de violencia conyugal.

\section{Procedimiento}

En primera instancia, se implementaron escenas videográficas, en donde se les presentaron a cada uno de los participantes cinco escenas que representaban violencia por parte de la mujer hacia el hombre. Dichas escenas fueron seleccionadas a partir de la concepción de Segato (2003) sobre la violencia conyugal; según ésta, el hombre, al salirse del modelo patriarcal, es en un principio la primera víctima del fenómeno, lo que genera consecuencias, como que la dinámica violenta de pareja sea imperceptible para ellos. Posteriormente, se revisaron los significados de las escenas proyectadas a través de la implementación de entrevistas semiestructuradas, en donde se amplió e indagó por el fenómeno de violencia como constructo cultural, sus formas, consecuencias y su comprensión desde las características de la dependencia afectiva. 


\section{Técnicas de recolección de datos}

Se implementó una entrevista semiestructurada, la cual tuvo en cuenta tres ámbitos del significado: cognitivo, comportamental y emocional. Lo anterior tuvo como fin conocer la construcción de significados de los participantes a través de sus pensamientos, acciones y sentimientos asociados a las experiencias, tanto propias como ajenas, de la violencia conyugal.

A continuación, se presentan las preguntas orientadoras que se diseñaron para cada ámbito. En primer lugar, en lo que respecta al ámbito cognitivo, tales preguntas fueron:

- ¿Qué pensamientos venían a su mente mientras observaba las escenas?

- ¿Qué imagen tiene usted de una mujer que violenta a un hombre?

- ¿Por qué cree usted que ese hombre violentado por su pareja continúa con ella?

- ¿Por qué cree usted que los hombres niegan y omiten ser violentados por sus parejas?

En segundo lugar, las preguntas orientadoras para el ámbito comportamental fueron:

- De acuerdo con las imágenes observadas, ¿Ha experimentado usted alguna vez una situación similar?

- ¿Qué ha hecho usted cuando ha experimentado cualquier tipo de violencia por parte de su pareja?

- ¿Cuál es su reacción frente a un caso donde una mujer le está gritando a un hombre en la calle?

- Si usted experimentara una situación similar, ¿Estaría dispuesto a comunicarla?

Finalmente, las preguntas orientadoras para el ámbito emocional fueron:

- ¿Qué sentimiento le produjeron las escenas observadas?

- ¿Cuál de las escenas le impacto más?

- ¿Qué sentimientos considera usted que llevan a que un hombre tolere una situación similar?

\section{Análisis de datos}

El análisis de datos se llevó a cabo mediante la implementación del software ATLAS.ti, en el cual, mediante la comparación de las entrevistas, se establecieron diferentes categorías semánticas de acuerdo con las expresiones individuales que tenían sentidos similares. De esta manera, los resultados se establecieron a partir de cinco categorías, en las cuales se presentaron las declaraciones de los participantes en primera persona, sintetizando el discurso colectivo.

\section{Resultados}

Los resultados evidenciaron que los hombres significan la violencia conyugal hacia el hombre como acciones físicas que se dan entre pareja, comportamientos que se toleran con la finalidad de ser digno de la misma, como una consecuencia de la dependencia afectiva y/o como un comportamiento de dominancia que genera burlas. Por otra parte, se encontró que la invisibilización de la violencia conyugal hacia el hombre tiene una alta relación con los patrones culturales acerca del rol masculino en la sociedad (ver figura 1). 


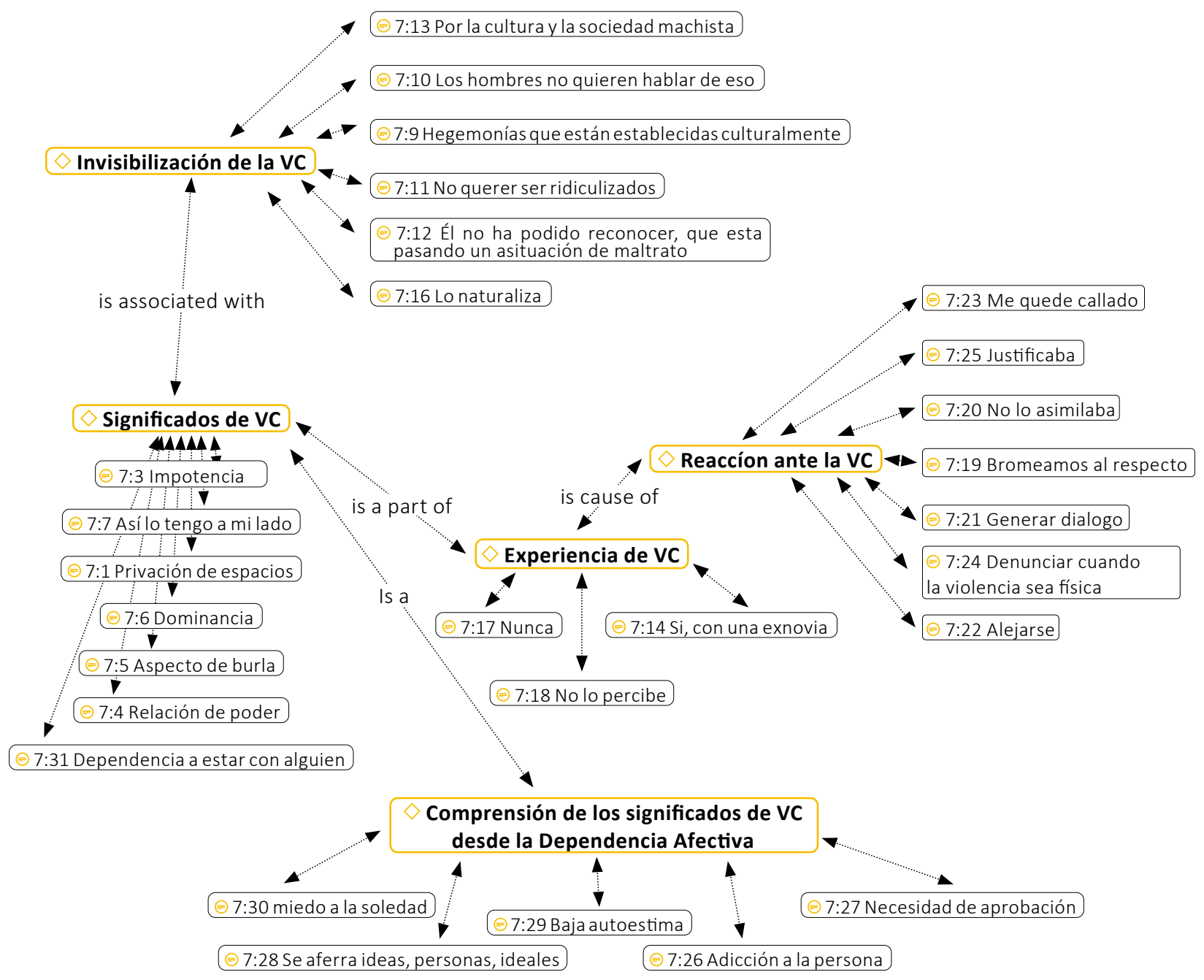

Figura 1. Red de significados de violencia conyugal

\section{Violencia conyugal}

Los significados que tuvieron los participantes respecto a la violencia hacia el género masculino se relacionaron generalmente con las relaciones de poder y dependencia (ver figura 2).

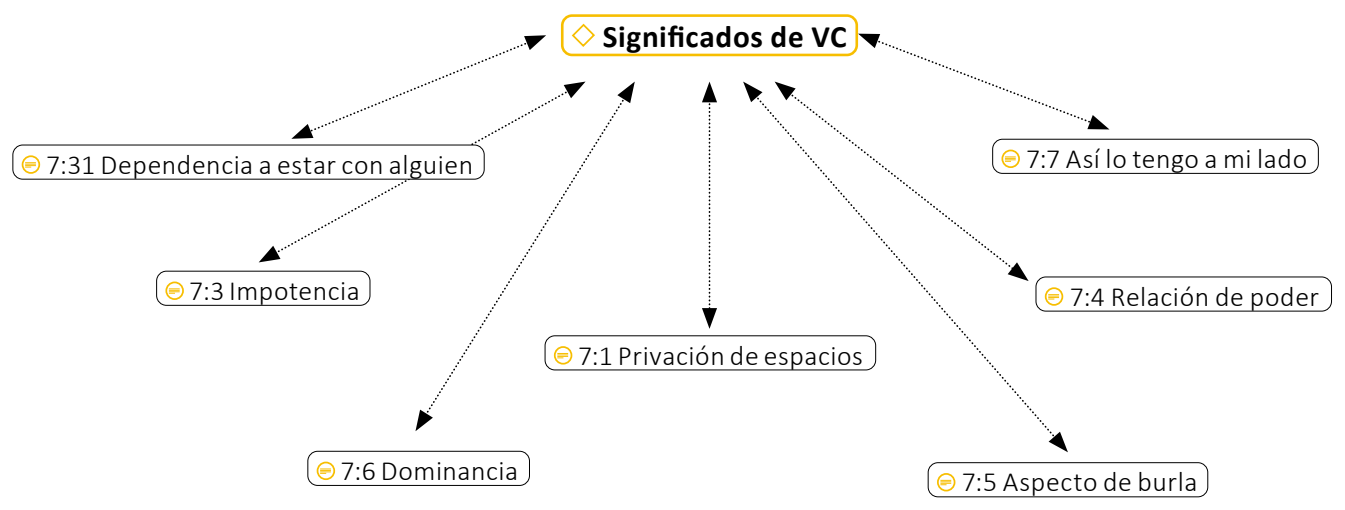

Figura 2. Significados de la violencia conyugal 
Los hombres se sienten impotentes, cuestionados, dominados y, algunas veces, identificados, lo que les genera rabia. Lo anterior se manifiesta en la siguiente intervención: "iQué bandera! Me daba rabia... sí, era como lo principal, porque este es como el tipo de maltrato que menos se evidencia. Un tipo de maltrato como la manipulación, privación de espacios, todo eso. Y en algún momento de mi vida lo viví; entonces, uno como que recuerda eso", S1. Los hombres paralelamente planteaban que la violencia conyugal es producto de la dependencia afectiva, como se pone de manifiesto en el siguiente ejemplo: "Cuando no hay amor propio, puede ser una persona que siente dependencia a estar con alguien, una necesidad de querer estar con alguien y no se siente bien estando solo y, de esta forma, acepta todo tipo de violencia",S2. Así mismo, los participantes argumentan que estos comportamientos se toleran con la finalidad de ser digno de la pareja, como se observa en la siguiente intervención: "Si hablamos de sentimientos negativos, necesidad de aprobación, necesito mostrarle al otro que soy digno de él, y lo demuestro dejando que me controle porque así lo tengo a mi lado", S4. Por otra parte, los participantes asumen que en una relación violenta existe una dinámica de dominancia y poder. Esto se ve claramente en el siguiente apartado: "Me hace cuestionar qué nivel de poder tiene esa mujer sobre ese hombre, porque obviamente hay una dominancia", S4. Finalmente, los estudiantes plantean que sufrir de violencia conyugal es un aspecto que genera burla e incredulidad. Lo anterior se puede observar en la siguiente intervención: "Es muy loco escuchar a un hombre, "no, es que me pegan en la casa" y más esa forma de burla, es hasta gracioso "uy, vean, le van a pegar en la casa"; entonces, empieza a ser como un aspecto de burla. Pura dramaturgia ahí prácticamente", S3. Por consiguiente, los hombres significan que la violencia hace parte de una dinámica de dominancia, en donde existen sentimientos de miedo y burla que llegan a generar dependencias afectivas.

\section{Invisibilización de la violencia conyugal}

La explicación del porqué los hombres no denuncian la violencia conyugal refiere principalmente al componente cultural y social que se tiene respecto al rol masculino (ver figura 3).

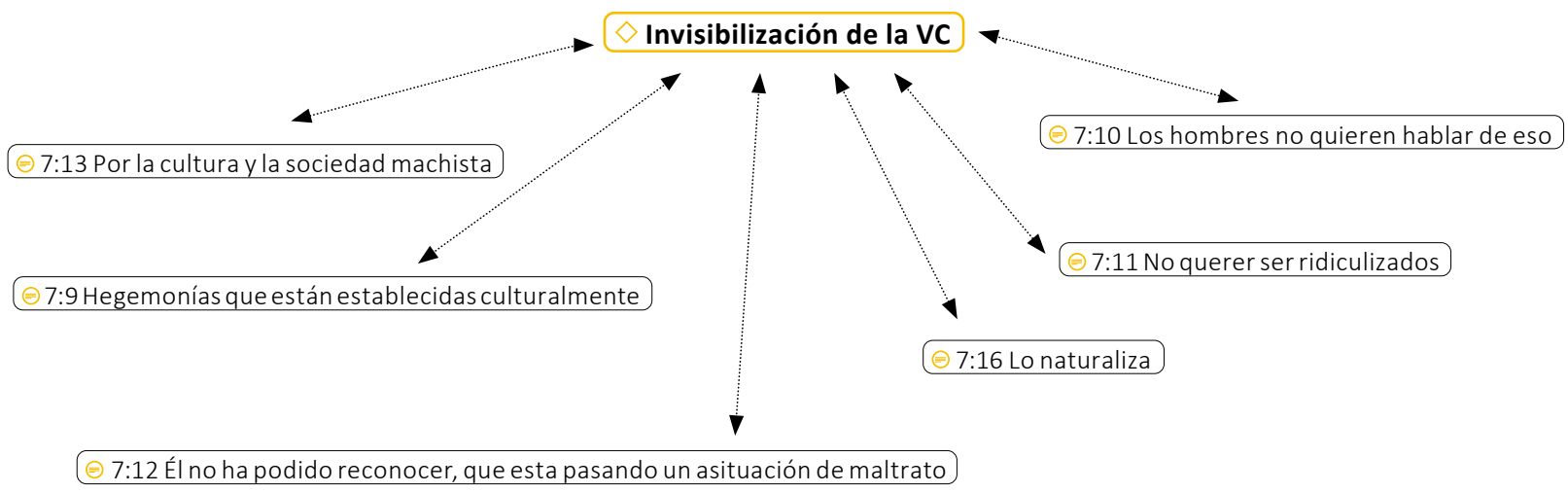

Figura 3. Invisibilización de la violencia conyugal

Existen unos roles establecidos culturalmente en los que, a través de la historia, se ha reflejado un falocentrismo, el cual posiciona a la mujer en un estado de debilidad y al hombre en el de la dominancia. Es así como los hombres deducen que no pueden manifestarse como víctimas de violencia, ya que para ellos los principales ejecutores son los hombres. Lo anterior se ratifica en el siguiente apartado: "Por esas hegemonías que están establecidas culturalmente, siempre están en pro de que la mujer es la víctima y el hombre, el victimario, por la fuerza, por el rol que siempre ha tenido culturalmente el hombre con el falocentrismo", S5. Por consiguiente, los hombres atribuyen a este fenómeno un aspecto de burla, lo que se pone de manifiesto en el siguiente comentario: "Porque si decimos que una mujer nos está pegando, lo más seguro es que nos van a 
juzgar, se van a burlar de nosotros, es decir, está mal visto que un hombre acepte que una mujer le está pegando", S2. Por la variedad de tipos de violencia, las personas, en este caso los hombres, tienden a no percibir las circunstancias que se enmarcan en lo psicológico; es así como la mayoría de los participantes están dispuestos a denunciarla solo cuando se trate de agresiones físicas. Lo anterior se corrobora con la siguiente opinión: "Estaría dispuesto a denunciar cuando la violencia sea física, pero si fuese psicológico, como manipulación, no lo denunciaría. Sin embargo, uno minimiza las cosas, porque como no ve el morado, no ve las consecuencias físicas, entonces uno normaliza", S1. En ese sentido, cabría resaltar que los roles establecidos culturalmente sobre qué es ser hombre y mujer en la sociedad tienen que ver con el reconocimiento o la invisibilidad del fenómeno por parte de sus víctimas.

\section{Experiencias de la violencia conyugal}

Los participantes declararon que no eran víctimas de violencia conyugal, estimando únicamente el componente físico del comportamiento (ver figura 4).

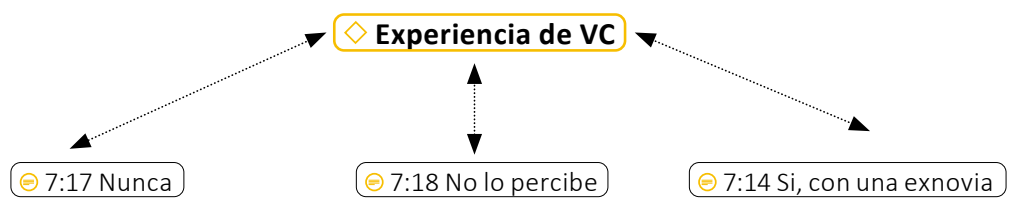

Figura 4. Experiencias de la violencia conyugal

Respecto a las experiencias de VC en hombres, los participantes comunicaron haber sido violentados en algún momento de su vida, como se observa a continuación: "De hecho sí, no ahorita, pero sí antes. En la escena; por ejemplo, que pasa otra chica y se arma la escena de celos, me ha pasado", S3. No obstante, existe un sesgo en cuanto a otros tipos de violencia que no sean físicos; por lo tanto, aunque los hombres manifiestan inicialmente no haberlo experimentado, en el transcurso de la entrevista, estos refieren que sí experimentaron algún tipo de violencia. Esto se demuestra en la siguiente intervención: "Ahora que recuerdo, en algún momento si lo experimenté; antes dije que no, pero ahora que recuerdo sí, tal vez no con la misma magnitud del video. Yo pienso también que ese maltrato psicológico es a la vez más silencioso y lo naturaliza uno", S2. Esto quiere decir que los hombres, al igual que las mujeres, suelen reconocer con mayor facilidad la violencia física y verbal, ignorando el carácter psicológico de las dinámicas violentas.

\section{Reacción ante la violencia conyugal}

Los comportamientos asumidos por los participantes ante una situación de violencia conyugal fueron diversos, pero podrían ser categorizados como comportamientos evasivos (ver figura 5).

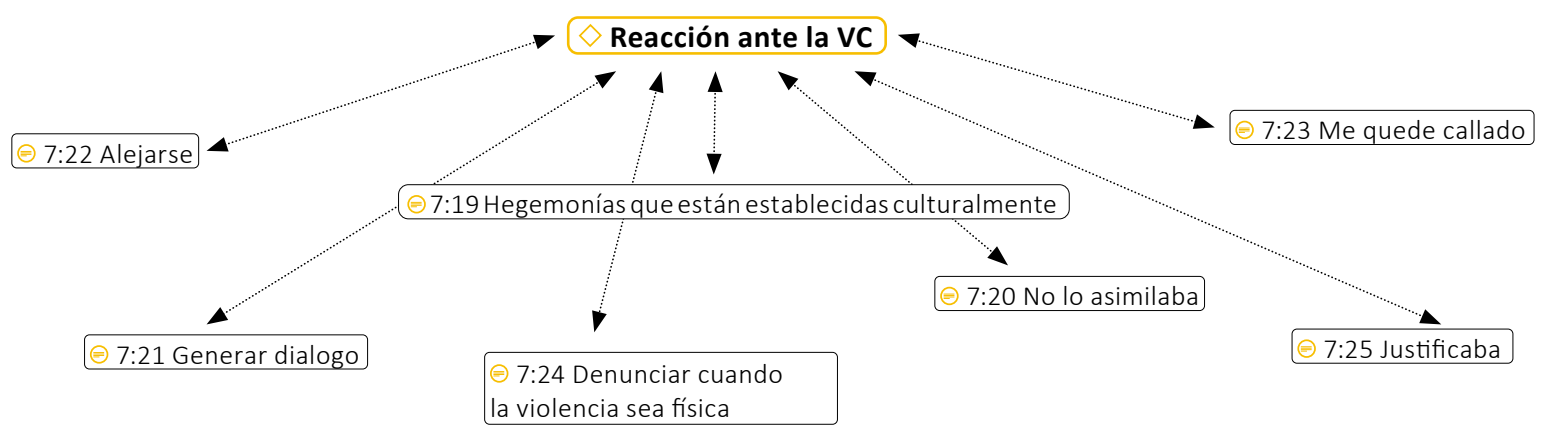

Figura 5. Reacción ante la violencia conyugal 
Frente a los actos de violencia conyugal, los participantes manifestaron una actitud neutral al principio, argumentando que no se debían involucrar en este tipo de conflictos o que deberían generar diálogos o llamadas a autoridades competentes. Esto se pone de manifiesto en el siguiente apartado: "La verdad en este tema de intervenir, trato de generar un diálogo; sin embargo, lo mejor es llamar a una autoridad competente porque de pronto uno al tratar de intervenir, también puede llegar a violentar o a someter su propia integridad física", S3. En contraste, también se pudo identificar una actitud de burla al respecto, como se observa a continuación: "Varias veces me he reído, pero también hay intenciones de tatar de separar y decirles que se alejen", S4. De esta manera, se podría establecer que, para los hombres, existe una ambivalencia respecto a lo que deberían hacer en un caso de violencia conyugal.

\section{La violencia conyugal comprendida desde la dependencia afectiva}

Los participantes resaltan que, en este tipo de conductas, las personas sienten un tipo de adicción a esta dinámica; así mismo, refieren apego y sugieren que los hombres se aferran a un ideal y, por eso, terminan involucrados en situaciones violentas de pareja. Esto se observa a continuación: "Siento que es por apego, lo que había mencionado anteriormente. Por ejemplo, si sientes apego por una persona, vas a permitir que la persona pase por encima de ti ... se siente aferrado, no quiere perder. Se aferra a una situación que puede cambiar, pero él no siente que sea fácil. Se aferra a ideas, personas, ideales; se convierte en un estilo de vida también", S1. Así mismo, se asociaron características de la dependencia afectiva con la baja autoestima y la necesidad de aprobación, como se aprecia en el siguiente comentario: "Si hablamos de sentimientos negativos, entonces la tolera porque hay una necesidad de aprobación, de mostrarle al otro que soy digno de él, dejando que me controle porque así lo tengo a mi lado", S4. Por último, los participantes refieren que la violencia es un comportamiento que se tolera en pareja con la finalidad de conservarla. Esto se observa claramente en el siguiente apartado: "Lo principal es que hay una falta de amor propio ahí y también la necesidad de no quedarse solo, como un miedo a la soledad", S2. Por lo tanto, de acuerdo con las características de la dependencia afectiva, tales como el miedo a la soledad, la baja autoestima y querer ser digno del otro, se evidenció que esta característica psicológica también se manifiesta en la dinámica de violencia conyugal (ver figura 6).

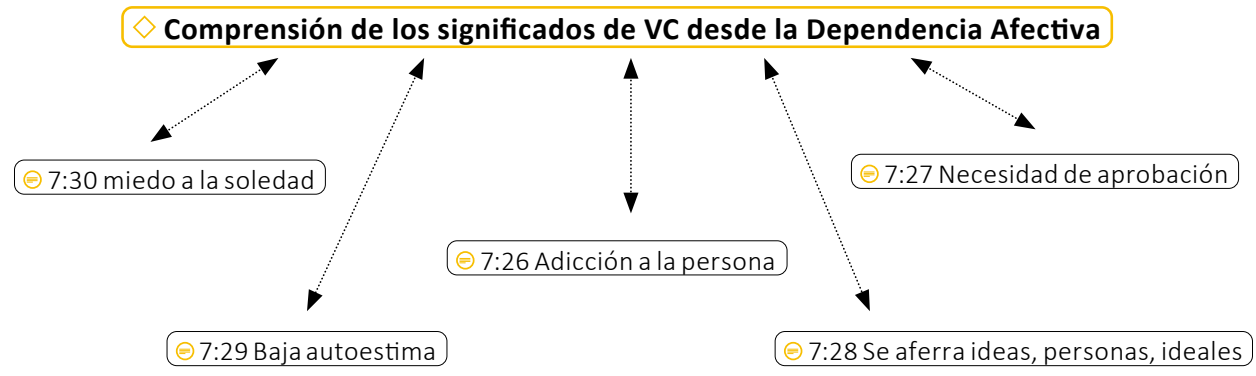

Figura 6. Comprensión de los Significados de VC desde la dependencia afectiva

\section{Discusión}

Colombia ha sufrido en los últimos 50 años una creciente violencia y violación de los derechos humanos (Pineda \& Otero, 2004). Dentro de la multiplicidad de violencia vivida, se encuentra la violencia conyugal, la cual ha sido señalada en algunos estudios como el padecimiento del género femenino (Ravazzola, 1997; Rojas-Andrade et al., 2013; Zancan \& Habigzang, 2018). No obstante, en contraposición, podemos encontrar algunas investigaciones que sugieren que la violencia conyugal también afecta a los hombres (Pacheco-Maldonado \& Castañeda-Figueroa, 2013; 
Trujano, Martínez \& Camacho 2010); así mismo, la variedad de investigaciones sobre violencia conyugal intenta explorar y conocer este fenómeno (Da Silva et al., 2018; Caldwell, Swan, \& Woodbrown, 2012; González \& Fernández, 2014; Ocampo \& Amar, 2011). En consecuencia, la presente investigación está interesada en comprender los significados de violencia conyugal hacia el hombre a través del concepto de dependencia afectiva como característica psicológica en seis hombres universitarios que pertenecen a una facultad de psicología.

De acuerdo con lo anterior, se identificó, al igual que en otras investigaciones sobre violencia conyugal, que los hombres significan la violencia como acciones físicas o verbales que se dan en la pareja; sin embargo, omiten el carácter psicológico de la violencia, lo cual podría indicar la falta de caracterización de la violencia y la invisibilidad de ésta para los hombres.

Paralelamente, los hombres significan que ser violentados por una mujer es sinónimo de debilidad y burla debido a aquellas hegemonías establecidas culturalmente sobre lo que es ser hombre en una sociedad machista. Ser hombre es sinónimo de fuerza y poder (Almonacid et al., 1996; Pineda, 2004), por lo que aquellos comportamientos que se salgan del prototipo de masculinidad pueden ser vistos con incredulidad y burla, lo que genera más juicios de valor que soluciones para la problemática. Según Segato (2003), cuando la pregunta sobre si se ha sufrido o no violencia conyugal es de carácter directo, la mayor parte del grupo de participantes responden negativamente, pero cuando se cambia el sentido de la cuestión y se muestran características específicas del maltrato, el número de víctimas se eleva. Esto denota el carácter normativo y asimilativo del fenómeno.

En consecuencia, la significación sobre la violencia conyugal como acción física y verbal, sumada a la percepción de que ser hombre y ser víctima es un aspecto de burla para la sociedad, pueden ser las características que conllevan a que la problemática sea más visible para la mujer que para el hombre. Según Pineda \& Otero (2004), lo anterior tiene relación con la trasformación social y política del rol de la mujer, lo cual le ha permitido tener en cuenta la importancia de su dignidad y; por lo tanto, ha podido sacar esta problemática de violencia del ámbito de lo privado. En ese sentido, al tener un rol estereotipado sobre la masculinidad, las mujeres pueden llegar a violentar a sus parejas a través de una dinámica silenciosa que no deja huellas, además de que es normalizada y genera burla. Según Ocampo (2011), esta modalidad de violencia es invisible, altamente nociva y, en la mayoría de ocasiones, pasa desapercibida. Por este motivo, las acciones violentas se naturalizan y minimizan, hasta el punto de convertirse en una dinámica convencional de pareja (Haack, Pressi \& Falcke, 2018; Lessard et al., 2015; Pacheco \& Castañeda, 2013).

Algunos estudios indican que la baja autoestima y la dependencia afectiva son algunos de los fenómenos psicológicos que pueden estar asociados a la violencia conyugal (Fernández, Welland \& Candelas, 2007; Rojas-Andrade et al., 2013). Por otra parte, Ocampo \& Amar (2011) afirman que no existen acuerdos en la comprensión de violencia conyugal, pero hay un consenso en la idea de que este es un fenómeno multicausal, en donde convergen aspectos de tipo psicológico, psicosocial, económico, cultural, familiar, personal, entre otros. Ahora bien, esta multicausalidad se pudo evidenciar en el discurso de los participantes de la presente investigación. Por una parte, expresaron que la violencia conyugal de la mujer hacia los hombres puede ser producto de la dependencia afectiva, debido a que se presentan características como una baja autoestima, idealización de la pareja, intentos por ser digno del otro y permitir todo tipo de comportamiento en pareja con la finalidad de no perderle. Sin embargo, los participantes pusieron énfasis en aspectos psicosociales y familiares, como evitar la desestructuración familiar y el cuestionamiento del rol masculino, la burla y las hegemonías culturales, que posicionan a la mujer como la víctima, lo que genera silencio por parte del hombre frente al fenómeno.

Teniendo en cuenta que la presente investigación pretendía comprender la violencia conyugal hacia el género masculino desde la perspectiva de la DA, se pudo evidenciar, como se mencionó anteriormente, que si bien existe la interpretación de la violencia conyugal como producto de la 
dependencia afectiva, también existen otras características psicosociales que pueden conllevar a una comprensión más amplia del fenómeno y que; por lo tanto, supusieron una limitación. En consecuencia, es necesario problematizar en lo referente a aspectos psicosociales y culturales a través de la dinámica familiar, el trabajo y los amigos, con la finalidad de conocer qué características están asociadas al rol masculino e identificar cómo esto interfiere en la VC.

En concordancia con lo mencionado, se hace un llamado a que se continúen realizando investigaciones respecto al tema, teniendo en cuenta que la VC es un fenómeno bidireccional y multicausal (Caetano, Ramisetty \& Field, 2005; Ocampo, 2018). Por tal motivo, es necesario que estos estudios se realicen con fines más exploratorios, que intenten indagar por las diferencias encontradas en las dinámicas de noviazgo, matrimonio y unión libre. Finalmente, es indispensable tener en cuenta que tanto hombres como mujeres son susceptibles de ser generadores y receptores de VC. En consecuencia, sería importante que futuros estudios no se limiten a indagar sobre la violencia relacionada con una orientación sexual específica; de hecho, se debería indagar sobre la VC vivida por individuos con diferentes orientaciones sexuales, dándole un enfoque de género; además, se podrían incluir diferentes tipos de población, así como diversos contextos sociales y niveles socioeconómicos.

\section{Referencias}

Aiquipa-Tello, J. (2015). Dependencia emocional en mujeres víctimas de violencia de pareja. Revista de Psicología, 33 (2),412-437. Recuperado de http://www.scielo.org. pe/pdf/psico/v33n2/a07v33n2.pdf

Almonacid, F., Daroch., C, Mena., P, Palma., C, Razeto., M., \& Zamora, E. (1996). Investigación social sobre violencia conyugal. Última Década (4), 1-17. Recuperado de http://www.redalyc.org/pdf/195/19500414.pdf

Amor, P., \& Echeburúa, E. (2010). Claves Psicosociales para la permanencia de la víctima en una relación de maltrato. Clínica Contemporánea, 1(2), 97-104. Recuperado de http://www.revistaclinicacontemporanea.org/archivos/cc2010v1n2a3.pdf

Ansara, D. L., \& Hindin, M. J. (2011). Psychosocial Consequences of Intimate Partner Violence for Women and Men in Canada. Journal of Interpersonal Violence, 26(8), 1628-1645. Recuperado de https://doi. org/10.1177/0886260510370600

Bornstein, R. F. (1993). The dependent personality. Nueva York: Guilford Press.

Bowlby, J. (1980). La pérdida afectiva. Buenos Aires: Paidós.

Bowlby, J. (2003). Vínculos afectivos: formación, desarrollo y pérdida. Madrid: Morata

Branden, N. (1995). Los seis pilares de la autoestima. Buenos Aires: Editorial Paidós.

Bruner, J. (1991). Actos de significado: más allá de la revolución cognitiva. Madrid: Alianza

Caetano, R., Ramisetty, S., \& Field, A. (2005). Unidirectional and Bidirectional Intimate Partner Violence Among White, Black, and Hispanic Couples in the United States. Violence and Victims, 20(4), 393-406. doi: 10.1891/0886-6708.20.4.393

Caldwell, J. E., Swan, S. C., \& Woodbrown, V. D. (2012). Gender Differences in Intimate Partner Violence Outcomes. Psychology of Violence, 2(1), 42-57. Recuperado de https://doi.org/10.1037/a0026296

Castelló, J. (2005). Dependencia Emocional: Características y Tratamiento. España: Alianza Editorial.

Da Silva, M. R., Freitas de Oliveira, J., Pereira, N., Matias, M., Matheus-Estrela, F., \& da Silva, H. M. (2018). Interface between conjugal violence and alcohol consumption by the partner. Revista Brasileira de Enfermagem, 71(5), 2109-2115. Recuperado de https://doi.org/10.1590/0034-7167-2017-0540

Duarte, E., Gómez, F., \& Carrillo, C. (2010), Masculinidad y hombre maltratador. ¿Pueden las creencias de hombres y mujeres propiciar violencia de género? Revista de Psicología, 19(2), 7-30. Recuperado de https://www.redalyc.org/articulo.o a?id=26416966001 
Dubugras, S., \& Guevara, B. (2013). Personalidade de mulheres vítimas de violencia doméstica: uma revisão sistemática da literatura. Contextos Clínicos, 6(2), 106-116. Recuperado de http://dx.doi. org/10.4013/ctc.2013.62.04

Espada, F., \& Torres, P. (1996). Violencia en casa. Madrid: Aguilar.

Fernández, T., Welland, C., \& Candelas, J. (2007). Varones, migración y violencia conyugal: un estudio comparativo sobre autoestima en mexicanos residentes en la frontera. Psicología y Salud, 17(1), 93-103. Recuperado de http://www.redalyc.o rg/pdf/291/29117111.pdf

Floyd-Aristizábal, D., Loaiza, S., Sierra, M., López, J. D., \& Ricaurte, A. (2016) Violencia de pareja contra el hombre en Cali, Colombia. Colombia Forense, 3(2), 33-40. Recuperado de https://revistas.ucc.edu. co/index.php/ml/article/view/1700

González, H., \& Fernández, T. (2014). Hombres violentados en la pareja: Jóvenes de Baja California, México. Culturales, 2(2), 129-155. Recuperado de http://www.redalyc. org/pdf/694/69432742006.pdf

Haack, K. R., Pressi, J., \& Falcke, D. (2018). Predictors of Marital Physical Violence: Personal and Relational Characteristics. Psico-USF, 23(2), 241-252. https://doi.org/10.1590/1413-82712018230205

Heise, L., \& García-Moreno, C. (2013). Comprender y abordar la violencia contra las mujeres. Violencia infligida por la pareja. Washington, D.C. EEUU: Organización Panamericana de la Salud. Recuperado de https://www.paho.org/hq/dmdocumen ts/2014/20184-ViolenciaPareja.pdf

Hirigoyen, M. (2006). Reseña de "Mujeres maltratadas. Los mecanismos de la violencia en la pareja". Anuario de Psicología, 37(1-2) 189-192. Recuperado de http://www.reda lyc.org/pdf/970/97012824017.pdf

Laca, F., \& Mejía, J. (2017). Dependencia emocional, consciencia del presente y estilos de comunicación en situaciones de conflicto con la pareja. Enseñanza e Investigación en Psicología, 22(1) 66-75. Recuperado de https://www.redalyc.org/articulo.oa?id=292 51161006

Lessard, G., Montminy, L., Lesieux, É., Flynn, C., Roy, V., Gauthier, S., \& Fortin, A. (2015). Les violences conjugales, familiales et structurelles: vers une perspective intégrative des savoirs. Enfances, Familles, Générations, 22, 1-26. Recuperado de https://doi.org/10.7202/1031116a

Lemos, M., \& Londoño, N. (2006). Construcción y validación del Cuestionario de Dependencia Emocional en Población Colombiana. Acta Colombiana de Psicología, 9(2), 127-140. Recuperado de http://www. redalyc.org/html/798/79890212/

López, F., Moral, J., Díaz, R., \& Cienfuegos, Y. (2013). Violencia en la pareja. Un análisis desde una perspectiva ecológica. Ciencia Ergo Sum, 20(1), 6-16. Recuperado de http://www.redalyc.org/ pdf/104/10425466009.pdf

Pacheco, K., \& Castañeda, J. G. (2013). Hombres receptores de violencia en el noviazgo. Avances en psicología, 21(2), 207-221. Recuperado de http://www.unife.ed u.pe/publicaciones/revistas/psicologia/2013/2/Karina-Pacheco.pdf

Packer, M. (1985). La investigación hermenéutica en el estudio de la conducta humana. American Psychologist, 40(10), 1-25. Recuperado de http://www.psicologiacultural.o rg/Pdfs/Traducciones/La\%20investigacion\%20hermeneutica.pdf

Segato, R. L. (2003). Las estructuras elementales de la violencia: contrato y estatus en la etiología de la violencia (334). Brasil: Universidad de Brasilia.

Moral, M., \& Sirvent, C. (2008). Dependencias sentimentales o afectivas: etiología, clasificación y evaluación. Revista Española de Drogodependencias, 33(2), 150-167. Recuperado de http://www.redalyc. org/pdf/284/28412891004.pdf

Ocampo, L., \& Amar, J. (2011). Violencia en la pareja, las caras del fenómeno. Salud Uninorte, 27 (1), 108-123. Recuperado de http://rcientificas.uninorte.edu.co/inde x.php/salud/article/viewArticle/1901/5829 
Ocampo, L. (2018). Violencia por parte del compañero (a) íntimo (a), una revisión teórica. Universitas Científica, 12(2), 41-45. Recuperado de https://revistas.upb.e du.co/index.php/universitas/article/view/8140

Perrone, R., \& Nannini, M., (2007). Violencia y abusos sexuales en la familia: una visión sistémica de las conductas sociales violentas. Buenos Aires, Argentina:Paidós. Recuperado de http://www.bibliopsi.org/ docs/carreras/electivas/ECFP/Intervenciones-Psicologico-Forenses-en-Disfunciones-y-Patologias-Familiares-Puhl/Perrone\%20y\%20Nannini\%20

Picó, M., Echeburúa, E., \& Martínez, M. (2008). Personality disorder symptoms in women as a result of chronic intimate male partner violence. Journal of Family Violence, 23, 577-588. Recuperado de https://link.springer.com/article/10.1007/s10896-008-9180-9

Pineda, J., \& Otero, L. (2004). Género, violencia intrafamiliar e intervención pública en Colombia. Revista de estudios sociales, 17, 19-31. Recuperado de http://www.redaly c.org/articulo.oa?id=81501703

Preciado, P., Torres-Cendales, N., \& Rey, A. (2012). Mujeres que finalizaron una relación maltratante: características de personalidad, psicopatológicas y sociodemográficas. Universitas Psychológica, 11(1), 43-54. Recuperado de http://www.scielo.org.pe/scie lo.php?script=sci_arttext\&pid=S0254-92472015000200007

Ravazzola, M. (1997). Historias infames: Los maltratos en las relaciones. Buenos Aires: Paidoìs.

Rojas-Andrade, R., Galleguillos, G., Miranda, P., \& Valencia, J. (2013). Los hombres también sufren. Estudio cualitativo de la violencia de la mujer hacia el hombre en el contexto de pareja. Revista Vanguardia Psicológica Clínica Teórica y Práctica, 3(2), 150-159. Recuperado de https://dialnet.unirioja.es/servlet/articulo?codigo $=4815152$

Schaeffer, B. (1998). ¿Es Amor o es adicción?. Barcelona: Apóstrof

Toldos, M. (2013). Hombres víctimas y mujeres agresoras. La cara oculta de la violencia entre sexos. Córdoba: Editorial Cántico.

Trujano, P., Martínez, A., \& Camacho, S. (2010). Varones víctimas de violencia doméstica: un estudio exploratorio acerca de su percepción y aceptación. Diversitas: Perspectivas en Psicología, 6 (2), 339354. Recuperado de http://www.redalyc.or g/articulo.oa?id=67915140010

Villegas, M., \& Sánchez, N. (2013). Dependencia emocional en un grupo de mujeres denunciantes de maltrato de pareja. Revista Textos \& Sentidos, 7, 10-29. Recuperado de http://biblioteca.ucp.edu.co/ojs/ index.php/textosysentidos/article/view/807

World Health Organization. (2011). Preventing intimate partner and sexual violence against women: taking action and generating evidence. Washington, DC, EEUU: Pan American Health Organization. Recuperado de https://apps.who.int/iris/bitstream/han dle/10665/44350/9789275716359_por.pdf

Young, J. (1999) Cognitive Therapy for Personality Disorders: a schema-focused approach. Sarasota: Professional Resource Press.

Zancan, N., \& Habigzang, L. F. (2018). Regulação Emocional, Sintomas de Ansiedade e Depressão em Mulheres com Histórico de Violência Conjugal. Psico-USF, 23(2), 253-265. Recuperado de https://doi. org/10.1590/1413-82712018230206 